\title{
Cross-Validation of the Turkish Version of the 28-Item Impact of Vision Impairment Profile Test
}

\author{
Neslihan Sinim Kahraman', Duygu Gulmez Sevim², Ayșe Öner ${ }^{{ }^{*}}$ \\ ${ }^{1}$ Ophthalmology Department, Acibadem Kayseri Hospital, Kayseri, Turkey \\ ${ }^{2}$ Ophthalmology Department of Erciyes University, Medical School, Kayseri, Turkey \\ Email: *ayseozoner@gmail.com
}

How to cite this paper: Kahraman, N.S., Sevim, D.G. and Öner, A. (2019) CrossValidation of the Turkish Version of the 28-Item Impact of Vision Impairment Profile Test. Open Journal of Ophthalmology, 9, 194-202.

https://doi.org/10.4236/ojoph.2019.94021

Received: October 23, 2019

Accepted: November 16, 2019

Published: November 19, 2019

Copyright $\odot 2019$ by author(s) and Scientific Research Publishing Inc. This work is licensed under the Creative Commons Attribution International License (CC BY 4.0).

http://creativecommons.org/licenses/by/4.0/ (c) $\underset{\mathrm{EY}}{\mathrm{EY}}$ Open Access

\begin{abstract}
Purpose: To translate the original 28-item Impact of Vision Impairment Profile into Turkish and to investigate its validity and reliability. Methods: Patients with no limitations to respond and affected by a chronic eye disease including retinitis pigmentosa (RP), age-related macular degeneration (ARMD) and diabetic macular edema which cause low vision were enrolled. The Turkish version of the IVI test was administered to all participants. The linguistic translation followed the international guidelines of forward and backward translation. 256 subjects who had a Snellen visual acuity of 6/12 or worse in the eye with best corrected visual acuity (BCVA) completed the Turkish version of the IVI-28 item. Psychometric evaluation of the Turkish IVI test involved the assessment of internal consistency, test-retest reliability, convergent and known-groups validity. Results: The mean $( \pm S D)$ age of the participants was $53.67 \pm 17.22$ years. There were 256 patients with one of the following conditions: 105 RP (41\%), 77 ARMD (30\%), 74 DME (29\%). Patients with lower visual acuity (VA) had lower index scores than those with higher VA $(p=0.001)$, which showed a sufficient responsiveness. Conclusion: Statistical analysis showed that Turkish version of the IVI-28 item is a valid and reliable instrument to measure vision-related quality of life (VRQoL) in patients with low vision.
\end{abstract}

\section{Keywords}

IVI Test, Quality of Life, Questionnaire Validation, Visual Function, Retinitis Pigmentosa, Age-Related Macular Degeneration, Diabetic Macular Edema

\section{Introduction}

According to the World Health Organization (WHO), the definition of quality 
of life is an individual's perception of their position in life in the context of the culture and value systems in which they live and in relation to their goals, expectations, standards and concerns [1]. Patient-centered quality of life instruments and studies which allow prioritizing the perception of the patients while supplying the health services, has drawn attention in ophthalmology as well as other disciplines. First, quality of life evaluations was performed to evaluate the success of cataract surgeries in the field of ophthalmology, followed by glaucoma, optic neuritis and more [2]. It is important that the instruments should be developed sensitively to the various cultural settings in which it is applied. Thus validiation of the tests is an important aspect of the assessments. However, there is no available psychometrically valid Turkish language VRQoL measure up to date and very few studies have assessed the impact of low vision in daily life of Turkish population.

Low vision is defined as the chronic visual impairment that deteriorates daily life and is not possible to be corrected by ordinary spectacles or contact lenses [3]. The Impact of Vision Impairment (IVI) questionnaire has been developed and validated at Centre for Eye Research Australia (CERA) which involves three vision-specific subscales including: "reading and accessing information", "mobility and independence" and "emotional well-being" and measures the impact of vision impairment on vision-related quality of life (VRQoL) [3] [4] [5]. The questionnaire involves 28 items with 3 - 4 response options of Likert scaling, ranging from not at all to a lot, also have the "don't do this for other reasons" response option for the item 1 - 15. CERA recommends that Rasch analysis is conducted on the raw IVI responses, thus we use the Rasch-scaled 28-item version of the IVI defined by Lamoureux et al. [3].

In this study, our aim was to determine the validity, reliability, and measurement characteristics of the Turkish-version of the IVI questionnaire in a set of Turkish patients with various retinal diseases. We have also determined the impact of retinitis pigmentosa (RP), age-related macular degeneration (ARMD) and diabetic macular edema (DME) on the questionnaire on VRQoL in Turkish patients.

\section{Materials and Methods}

\section{Development of the Turkish-version of the IVI questionnaire}

The IVI questionnaire consists 28 items with three specific subscales: reading and accessing information, mobility and independence, and emotional well-being (Table 1). The response options for each item is based on a scale of 3 (not at all), 2 (a little), 1 (moderately) to 0 (a lot), with additional response choice of not applicable (don't do this activity for other reasons) for the item 1-15. Translation guidelines of WHO for research instruments were used for the development of the Turkish-version of IVI questionnaire and forward and backward translation was performed [6]. Pilot-testing of the cognitive debriefing test with ten Turkish ophthalmic patients were then assessed to evaluate their understanding and in- 
terpretation of the questionnaire and, proved to be well accepted and easy to understand. We adapted one question only according to the Turkish lifestyle and culture and slightly modified the question for the recreational activities icluding "bowling, walking or golf" as "walking and similar outdoor activities". The final version of the Turkish-version IVI questionnaire is outlined after the modifications (Table 1).

Table 1. Mean scores of all items of IVI in all subjects.

\begin{tabular}{|c|c|c|c|c|c|}
\hline & $\begin{array}{c}\mathrm{RP} \\
\text { Mean } \pm \mathrm{SD}\end{array}$ & $\begin{array}{c}\text { ARMD } \\
\text { Mean } \pm \text { SD }\end{array}$ & $\begin{array}{c}\text { DME } \\
\text { Mean } \pm \text { SD }\end{array}$ & $\begin{array}{c}\text { Control } \\
\text { Mean } \pm \text { SD }\end{array}$ & $p$ value \\
\hline 1. (R) Looking after appereance & $1.26 \pm 1.11$ & $1.77 \pm 1.13$ & $2.20 \pm 1.10$ & $3.0 \pm 0.0$ & $P<0.05$ \\
\hline 2. (R) Opening package & $1.26 \pm 1.08$ & $1.74 \pm 1.11$ & $2.11 \pm 0.96$ & $3.0 \pm 0.0$ & $P<0.05$ \\
\hline 3. (R) Getting information & $2.16 \pm 0.86$ & $1.77 \pm 0.94$ & $2.16 \pm 0.86$ & $3.0 \pm 0.0$ & $P<0.05$ \\
\hline 4. (R) Handling money & $1.30 \pm 1.08$ & $1.38 \pm 1.11$ & $2.03 \pm 1.05$ & $3.0 \pm 0.0$ & $P<0.05$ \\
\hline 5. (M) Recreational activities & $1.30 \pm 1.08$ & $1.27 \pm 1.07$ & $1.93 \pm 1.13$ & $3.0 \pm 0.0$ & $P<0.05$ \\
\hline 6. (M) Getting outdoors in familiar environments & $1.29 \pm 1.12$ & $1.37 \pm 1.04$ & $1.94 \pm 1.13$ & $3.0 \pm 0.0$ & $P<0.05$ \\
\hline 7. (M) Getting outdoors in unfamiliar environments & $1.64 \pm 1.21$ & $0.98 \pm 1.21$ & $1.54 \pm 1.23$ & $3.0 \pm 0.0$ & $P<0.05$ \\
\hline 8. (M) Travelling or using transport & $1.35 \pm 1.13$ & $1.07 \pm 1.22$ & $1.54 \pm 1.20$ & $3.0 \pm 0.0$ & $P<0.05$ \\
\hline 9. (M) Walking on uneven ground & $1.66 \pm 1.19$ & $1.04 \pm 1.09$ & $1.67 \pm 1.10$ & $3.0 \pm 0.0$ & $P<0.05$ \\
\hline 10. (M) Crossing the street & $1.44 \pm 1.24$ & $1.00 \pm 1.07$ & $1.66 \pm 1.10$ & $3.0 \pm 0.0$ & $P<0.05$ \\
\hline 11. (M) Safety at home & $1.20 \pm 1.21$ & $2.06 \pm 1.36$ & $2.47 \pm 0.87$ & $3.0 \pm 0.0$ & $P<0.05$ \\
\hline 12. (M) Spilling or breaking things & $1.34 \pm 1.01$ & $2.15 \pm 1.30$ & $2.52 \pm 0.80$ & $3.0 \pm 0.0$ & $P<0.05$ \\
\hline 13. (M) Burning or scalding yourself & $1.62 \pm 1.10$ & $1.87 \pm 1.12$ & $2.52 \pm 1.15$ & $3.0 \pm 0.0$ & $P<0.05$ \\
\hline 14. (M) Having a fall & $1.27 \pm 0.96$ & $1.79 \pm 1.04$ & $2.50 \pm 1.15$ & $3.0 \pm 0.0$ & $P<0.05$ \\
\hline 15. (M) Safety outside the home & $1.50 \pm 1.13$ & $1.47 \pm 1.14$ & $2.39 \pm 1.65$ & $3.0 \pm 0.0$ & $P<0.05$ \\
\hline 16. (M) Going down steps, stairs, or curb & $1.30 \pm 1.03$ & $1.36 \pm 1.11$ & $1.92 \pm 1.42$ & $3.0 \pm 0.0$ & $P<0.05$ \\
\hline 17. (E) Felt embarrassed & $1.66 \pm 1.25$ & $1.72 \pm 1.19$ & $1.73 \pm 1.19$ & $3.0 \pm 0.0$ & $P<0.05$ \\
\hline 18. (E) Felt frustrated or annoyed & $1.04 \pm 1.11$ & $1.26 \pm 1.21$ & $1.42 \pm 1.10$ & $3.0 \pm 0.0$ & $P<0.05$ \\
\hline 19. (E) Felt lonely or isolated & $1.70 \pm 1.23$ & $1.45 \pm 1.23$ & $1.61 \pm 1.15$ & $3.0 \pm 0.0$ & $P<0.05$ \\
\hline 20. (E) Felt sad or low & $0.90 \pm 1.10$ & $1.09 \pm 1.10$ & $1.38 \pm 1.13$ & $3.0 \pm 0.0$ & $P<0.05$ \\
\hline 21. (E) Worried about eyesight & $1.13 \pm 1.10$ & $1.60 \pm 1.04$ & $2.05 \pm 0.84$ & $3.0 \pm 0.0$ & $P<0.05$ \\
\hline 22. (E) Interference with the relationships with family & $1.62 \pm 1.10$ & $2.06 \pm 0.89$ & $2.31 \pm 0.77$ & $3.0 \pm 0.0$ & $P<0.05$ \\
\hline 23. (E) Felt like a nuisance or a burden & $2.07 \pm 1.23$ & $1.85 \pm 0.98$ & $2.14 \pm 0.83$ & $3.0 \pm 0.0$ & $P<0.05$ \\
\hline 24. (E) Felt vulnerable & $1.34 \pm 1.25$ & $1.89 \pm 0.87$ & $2.19 \pm 0.85$ & $3.0 \pm 0.0$ & $P<0.05$ \\
\hline 25. (E) Stopped doing something & $1.21 \pm 1.22$ & $1.66 \pm 0.96$ & $1.81 \pm 1.01$ & $3.0 \pm 0.0$ & $P<0.05$ \\
\hline 26. (E) Needed help from other people & $1.22 \pm 0.97$ & $1.53 \pm 1.00$ & $1.91 \pm 0.95$ & $3.0 \pm 0.0$ & $P<0.05$ \\
\hline 27. (E) Treated in the wrong way & $1.15 \pm 0.91$ & $1.87 \pm 0.90$ & $2.08 \pm 0.86$ & $3.0 \pm 0.0$ & $P<0.05$ \\
\hline 28. (E) Interfered with life in general & $1.20 \pm 1.06$ & $1.55 \pm 0.88$ & $1.73 \pm 1.04$ & $3.0 \pm 0.0$ & $P<0.05$ \\
\hline
\end{tabular}

IVI, Impact of Vision Impairment; R, reading and accessing information; M, mobility and independence; E, emotional well-being. RP: Retinitis pigmentosa; ARMD: Age-related macular degeneration; DME: Diabetic macular edema. Data are expressed as mean $\pm \mathrm{SD}$. ${ }^{*}$ Although the mean scores of the patients showed no difference between groups, the scores of all groups were significantly lower than the controls $(p<0.05)$. 


\section{Subjects}

226 subjects who admitted to our center between April 2019 and July 2019 were recruited in this prospective study. Inclusion criteria were: 1) Snellen visual acuity of $6 / 12$ or worse in the eye with best corrected visual acuity (BCVA) 2) A clinical diagnosis of a retinal disease including RP, ARMD and DME confirmed by optical coherence tomography and/or visual field test 3) Age older than 18 years.

Informed consent was obtained from each individual participant and the study was carried out with approval from the Institutional Review Board and adhered to the tenets of the Declaration of Helsinki. Sample size was set at minimum 70 subjects per group according to the statistical calculations [7]. In addition, 30 healthy volunteered individuals from the medical staff with no chronic ocular condition and $\geq 20 / 40$ BCVA in the better seing eye was enrolled for the control group. The exclusion criteria for all the participants were illiteracy or cognitive dysfunction. First, all 256 participants were asked to complete the questionnaire by themselves followed by a repeated questionnaire fill up in the following 4 - 7 weeks by 70 patients with RP to test the test-retest reliability.

\section{Statistical analysis}

For the validity of the questionnaire, the Rasch analysis was performed as suggested by the developers of the questionnaire. Differences between groups were evaluated using analysis of variance (ANOVA) and paired t-test. A $p$ value less than 0.05 was significant. The correlation between items was measured by Pearson's correlation coefficient and the internal consistency was analyzed by the Cronbach's alpha coefficient.

\section{Results}

A total of 256 people, ranging in age from 18 to 88 years old (mean \pm SD, 53.67 \pm 17.22 years), participated in the study and $44.1 \%(\mathrm{n}=113)$ were women (Table 2). Besides, 30 participants were enrolled as normal control (VA levels of 20/20 - 20/25 in the better eye). The 256 patients with a series of common retinal diseases were recruited and subdivided into 3 subject groups including one of the following conditions: 105 RP (41\%), 77 ARMD (30\%), 74 DME (29\%). The demographic characteristics of the patients were given in Table 2.

Table 2. The demographic characteristics of participants.

\begin{tabular}{ccccc}
\hline & RP & ARMD & DME & Control \\
\hline Number & 105 & 77 & 74 & 30 \\
Age (Years) & $35.7(18-49)$ & $67.3(58-88)$ & $62.7(54-78)$ & $32.4(28-46)$ \\
Gender (F/M) & $46 / 59$ & $33 / 44$ & $34 / 40$ & $15 / 15$ \\
Visual Acuity & $0.22 \pm 0.17$ & $0.18 \pm 0.16$ & $0.25 \pm 0.18$ & $0.99 \pm 0.02$ \\
\hline
\end{tabular}

RP: Retinitis pigmentosa; ARMD: Age-related macular degeneration; DME: Diabetic macular edema. *Although the mean visual acuity values of the patients showed no difference between groups, the values of all groups were significantly lower than the controls $(p<0.05)$. 
In all groups, no difficulty was observed in filling or understanding the questionnaire.

The composite and all subscales scores were significantly lower in all vision impairment groups compared with normal group ( $p$ value $<0.05$ ). The Cronbach alpha coefficient ranged from 0.80 to 0.92 for the subscales. Patients with lower levels of VA had significantly lower subscale scores than those with higher VA ( $p$ value $<0.05)$.

The mean scores of each IVI items for the different vision defects groups are all listed in Table 1. Lower values indicate lower visual ability and suggest that the subject is more disabled. The analysis on each subscale score among the three vision defects subgroups revealed no significant differences in the scores of either "mobility and independence", "emotional well being" or "reading and accessing information" between groups. But the scores of all three groups were significantly lower than the controls (Table 3$)(p<0.05)$.

\section{Discussion}

Retinitis pigmentosa (RP) is severe retinal neurodegeneration characterized with progressive loss of photoreceptors that leads to initial night blindness, consecutive tunnel vision and eventual total blindness [8]. It consists of a group of inherited disorders with dominant and recessive forms and linked to more than 40 genes which make the targeted therapies complicated [9].

Age-related macular degeneration is one of the leading causes of vision loss in the elderly population in developed countries [10]. Its main symptom is progressive and irreversible loss of central vision. The disease affects $8.7 \%$ of the elderly and the prevalence is expected to increase in the coming years [11]. In the last decade, numerous developments have been achieved in the treatment of wet AMD which have shown promising outcomes. However, the only approved treatment for dry AMD to date is AREDS combination which is used for delaying the progression of AMD to advanced form [12]. The effects of both early and late AMD on QoL have been assessed in detailed previously in the literature [13] [14] [15] [16] [17]. The disease is associated with little functional loss at its early stages however as it progresses to atrophic or neovascular late-stage AMD the prognosis is poor due to severely impaired central vision and the presence of central scotoma. It impacts the daily life of these patients on a variety of aspects

Table 3. Subscale scores for the normal controls and three patient groups.

\begin{tabular}{cccccc}
\hline & RP & ARMD & DME & Control & $p$ value \\
\hline $\begin{array}{c}\text { Reading and accessing } \\
\text { information }\end{array}$ & $13.65 \pm 7.63$ & $14.4 \pm 10.0$ & $19.4 \pm 9.4$ & 30.0 & $<0.05^{\star}$ \\
Mobility and independence & $8.23 \pm 4.16$ & $10.1 \pm 5.5$ & $13.31 \pm 5.57$ & 18.0 & $<0.05^{\star}$ \\
Emotional well-being & $16.14 \pm 9.27$ & $19.53 \pm 9.56$ & $22.36 \pm 9.37$ & 36.0 & $<0.05^{\star}$ \\
\hline
\end{tabular}

Data are expressed as mean \pm SD. RP: Retinitis pigmentosa; ARMD: Age-related macular degeneration; DME: Diabetic macular edema. ${ }^{\star}$ Although the mean scores of the patients showed no difference between groups, the scores of all groups were significantly lower than the controls. 
including their ability to watch television, read, face recognition and doing manual work that requires near central vision [18]. The deterioration of vision in AMD has also been associated with emotional status, falls and mobility [19] [20]. As for estimating the impact of AMD on QoL, Slakter and Stur [21] suggested that it is more important to consider a patient's ability to adapt and cope other than considering the degree of vision loss in isolation which highlights the importance of measuring the VRQoL in patients with low vision with reliable tests.

Diabetic macular edema is a vision threatening complication of diabetes mellitus (DM) in working-age adults [22] [23]. DME can occur at any stage of diabetic retinopathy and is responsible for severe central vision loss [24]. Diabetic ocular complications are also known to have impacts on QoL from preliminary symptoms to severe vision loss [25]. Assesment of patients' QoL has been implemented to determine the outcomes of clinical therapeutic decisions. The 25-item National Eye Institute Visual Function Questionnaire (NEI VFQ-25) has been used in various phase 3 trials to evaluate the efficacy and safety of intravitreal anti-VEGF therapy in patients with DME and visual acuity improvement in patients with DME treated with anti-VEGF agents has been found to be correlated with enhanced patient-reported visual function measured with NEI VFQ-25 [26] [27] [28].

Vision impairment puts a huge burden on the individuals, society and economy. Up to date, many studies have revealed that impaired vision reflects highly on quality of life. Recent developments on the treatment of retinal degenerative and vascular diseases have gained popularity in the field with promising and encouraging results and have been a hope for the patients with "irreversible" vision loss to restore their visual function to some level. It is not optimal to only evaluate the success of these fairly new treatment options with vision improvement only, rather it is more important to determine the impact of the treatment on the VRQoL [29]. For instance, in the Reticell-clinical trial the investigators assessed the VRQoL in patients with RP submitted to intravitreal use of bone marrow-derived stem cells with National Eye Institute Visual FunctionQuestionnaire-25 (NEI VFQ-25) [9]. The outcomes of the study showed that the cell therapy with intravitreal use of bone marrow-derived stem cells could improve the quality of life of patients with RP, although the improvement was lost with time. In their report Seo et al. suggested that visual quality cannot be explained only by visual acuity or visual fields in RP patients, rather vision-specific quality of life can be explained by both visual acuity and visual field in RP patients [30].

When determining the impact of low vision on individuals, it is important to quantify its impact on these individuals' daily life and activities. Besides, evaluating the VRQoL of individuals with socioeconomic income living in developing countries need to have the aspects of being cheap, easily available and reliable [31]. The results of our study showed that the Turkish-version of the IVI questionnaire is a reliable and valid tool to measure VRQoL in patients with low vision of various underlying conditions. The validity of the questionnaire was 
determined according to the ability of the Turkish-version IVI to detect vision impaired individuals and differentiate them from healthy participants. The subscales of the questionnaire also revealed high internal consistencies and high test-retest reliability in the test-retest subgroup of the patients and controls.

The VRQoL has been shown to be determined mainly by the level of vision loss as well as the type of visual field loss (peripheral versus central, and so forth) irrespective of the underlying condition [20]. In our set of patients, we found that all the questions were able to distinguish between the groups which we think is mainly due to the difference in the type of visual field loss between the underlying conditions.

\section{Conclusion}

In conclusion, the outcome of this study indicates that the symptoms of common retinal diseases are associated with an adverse impact on VRQoL and the Turkish-version of IVI questionnaire is able to evaluate the VRQoL in Turkish patients. Furthermore, it puts a relevant alternative to evaluate the treatment outcomes of novel treatment options in retinal conditions.

\section{Conflicts of Interest}

The authors declare no conflicts of interest regarding the publication of this paper.

\section{References}

[1] (1993) Study Protocol for the World Health Organization Project to Develop a Quality of Life Assessment İnstrument (WHOQOL). Quality of Life Research, 2, 153-159. https://doi.org/10.1007/BF00435734

[2] Idil, A., Ozen, M., Atak, N., et al. (2011) Validity and Reliability Study of Turkish Version on Low Vision with Quality of Life Questionnaire. International Journal of Ophthalmology, 4, 546-551.

[3] Lamoureux, E.L., Pallant, J.F., Pesudovs, K., et al. (2006) The Impact of Vision Impairment Questionnaire: An Evaluation of İts Measurement Properties Using Rasch Analysis. Investigative Ophthalmology \& Visual Science, 47, 4732-4741. https://doi.org/10.1167/iovs.06-0220

[4] Center for Eye Research Australia. Patient-Reported Outcome Questionnaires. https://www.cera.org.au/pro-questionnaires

[5] Weih, L.M., Hassell, J.B. and Keeffe, J. (2002) Assessment of the İmpact of Vision İmpairment. Investigative Ophthalmology \& Visual Science, 43, 927-935.

[6] Sartorius, N. and Kuyken, W. (1994) Translation of Health Status İnstruments. In: Quality of Life Assessment. International Perspectives, Springer, Berlin, 3-18. https://doi.org/10.1007/978-3-642-79123-9 1

[7] Tabachnick, B.G., Fidell, L.S. and Ullman, J.B. (2007) Using Multivariate Statistics. Pearson, Boston.

[8] Oner, A., Gonen, Z.B., Sinim, N., et al. (2016) Subretinal Adipose Tissue-Derived Mesenchymal Stem Cell İmplantation in Advanced Stage Retinitis Pigmentosa: A Phase I Clinical Safety Study. Stem Cell Research \& Therapy, 7, 178. 
https://doi.org/10.1186/s13287-016-0432-y

[9] Siqueira, R.C., Messias, A., Messias, K., et al. (2015) Quality of Life in Patients with Retinitis Pigmentosa Submitted to İntravitreal Use of Bone Marrow-Derived Stem Cells (Reticell-Clinical Trial). Stem Cell Research \& Therapy, 6, 29. https://doi.org/10.1186/s13287-015-0020-6

[10] Mousavi, S.A., Kouchari, M.R., Samdani-Fard, S.H., et al. (2012) Relationship between Serum Levels of Testosterone and the Severity of Chronic Obstructive Pulmonary Disease. Tanaffos, 11, 32-35.

[11] Wong, W.L., Su, X., Li, X., et al. (2014) Global Prevalence of Age-Related Macular Degeneration and Disease Burden Projection for 2020 and 2040: A Systematic Review and Meta-Analysis. The Lancet Global Health, 2, e106-e116. https://doi.org/10.1016/S2214-109X(13)70145-1

[12] Age-Related Eye Disease Study 2 Research Group (2013) Lutein + Zeaxanthin and Omega-3 Fatty Acids for Age-Related Macular Degeneration: The Age-Related Eye Disease Study 2 (AREDS2) Randomized Clinical Trial. JAMA, 309, 2005-2015. https://doi.org/10.1001/jama.2013.4997

[13] Lamoureux, E.L., Mitchell, P., Rees, G., et al. (2011) Impact of Early and Late Age-Related Macular Degeneration on Vision-Specific Functioning. British Journal of Ophthalmology, 95, 666-670. https://doi.org/10.1136/bjo.2010.185207

[14] Wong, E.Y., Guymer, R.H., Hassell, J.B., et al. (2004) The Experience of Age-Related Macular Degeneration. Journal of Visual Impairment \& Blindness, 98, 629-640. https://doi.org/10.1177/0145482X0409801007

[15] Espallargues, M., Czoski-Murray, C.J., Bansback, N.J., et al. (2005) The İmpact of Age-Related Macular Degeneration on Health Status Utility Values. Investigative Ophthalmology \& Visual Science, 46, 4016-4023. https://doi.org/10.1167/iovs.05-0072

[16] Finger, R.P., Fenwick, E., Marella, M., et al. (2011) The İmpact of Vision İmpairment on Vision-Specific Quality of Life in Germany. Investigative Ophthalmology \& Visual Science, 52, 3613-3619. https://doi.org/10.1167/iovs.10-7127

[17] Mangione, C.M., Gutierrez, P.R., Lowe, G., et al. (1999) Influence of Age-Related Maculopathy on Visual Functioning and Health-Related Quality of Life. American Journal of Ophthalmology, 128, 45-53. https://doi.org/10.1016/S0002-9394(99)00169-5

[18] Birk, T., Hickl, S., Wahl, H.W., et al. (2004) Development and Pilot Evaluation of a Psychosocial İntervention Program for Patients with Age-Related Macular Degeneration. Gerontologist, 44, 836-843. https://doi.org/10.1093/geront/44.6.836

[19] Mackenzie, P.J., Chang, T.S., Scott, I.U., et al. (2002) Assessment of Vision-Related Function in Patients with Age-Related Macular Degeneration. Ophthalmology, 109, 720-729. https://doi.org/10.1016/S0161-6420(01)01021-1

[20] Finger, R.P., Fenwick, E. and Lamoureux, E.L. (2013) Impact of Early and Late Age-Related Macular Degeneration on Quality of Life. In: Ophthalmology and the Ageing Society, Springer, Berlin, 181-192. https://doi.org/10.1007/978-3-642-36324-5 12

[21] Slakter, J.S. and Stur, M. (2005) Quality of Life in Patients with Age-Related Macular Degeneration: İmpact of the Condition and Benefits of Treatment. Survey of Ophthalmology, 50, 263-273. https://doi.org/10.1016/j.survophthal.2005.02.007

[22] Romero-Aroca, P. (2010) Targeting the Pathophysiology of Diabetic Macular Edema. Diabetes Care, 33, 2484-2485. https://doi.org/10.2337/dc10-1580 
[23] Romero-Aroca, P., Baget-Bernaldiz, M., Pareja-Rios, A., et al. (2016) Diabetic Macular Edema Pathophysiology: Vasogenic versus İnflammatory. Journal of Diabetes Research, 2016, Article ID: 2156273. https://doi.org/10.1155/2016/2156273

[24] Fenwick, E.K., Khadka, J., Pesudovs, K., et al. (2017) Diabetic Retinopathy and Macular Edema Quality-of-Life Item Banks: Development and Initial Evaluation Using Computerized Adaptive Testing. Investigative Ophthalmology \& Visual Science, 58, 6379-6387. https://doi.org/10.1167/iovs.16-20950

[25] Loftus, J.V., Sultan, M.B., Pleil, A.M., et al. (2011) Changes in Vision- and Health-Related Quality of Life in Patients with Diabetic Macular Edema Treated with Pegaptanib Sodium or Sham. Investigative Ophthalmology \& Visual Science, 52, 7498-7505. https://doi.org/10.1167/iovs.11-7613

[26] Granstrom, T., Forsman, H., Leksell, J., et al. (2015) Visual Functioning and Health-Related Quality of Life in Diabetic Patients about to Undergo Anti-Vascular Endothelial Growth Factor Treatment for Sight-Threatening Macular Edema. Journal of Diabetes and Its Complications, 29, 1183-1190. https://doi.org/10.1016/j.jdiacomp.2015.07.026

[27] Korobelnik, J.F., Do, D.V., Schmidt-Erfurth, U., et al. (2014) Intravitreal Aflibercept for Diabetic Macular Edema. Ophthalmology, 121, 2247-2254.

https://doi.org/10.1016/j.ophtha.2014.05.006

[28] Garweg, J.G., Stefanickova, J., Hoyng, C., et al. (2019) Vision-Related Quality of Life in Patients with Diabetic Macular Edema Treated with Intravitreal Aflibercept: The AQUA Study. Ophthalmol Retina, 3, 567-575. https://doi.org/10.1016/j.oret.2019.03.012

[29] Oner, A. (2018) Stem Cell Treatment in Retinal Diseases: Recent Developments. Turkish Journal of Ophthalmology, 48, 33-38. https://doi.org/10.4274/tjo.89972

[30] Seo, J.H., Yu, H.G. and Lee, B.J. (2009) Assessment of Functional Vision Score and Vision-Specific Quality of Life in İndividuals with Retinitis Pigmentosa. Korean Journal of Ophthalmology, 23, 164-168. https://doi.org/10.3341/kjo.2009.23.3.164

[31] Ratanasukon, M., Tongsomboon, J., Bhurayanontachai, P., et al. (2016) The Impact of Vision Impairment (IVI) Questionnaire; Validation of the Thai-Version and the Implementation on Vision-Related Quality of Life in Thai Rural Community. PLoS ONE, 11, e0155509. https://doi.org/10.1371/journal.pone.0155509 